\title{
Prediction Model of Defective Areas of Gas Pipeline Through Law Given Weibull Distribution
}

\author{
Maria Sergeevna Danilenko \\ Samara National Research University \\ Samara university \\ Samara, Russian Federation
}

\author{
Sergey Antonovich Prokhorov \\ Samara National Research University \\ Samara university \\ Samara, Russian Federation
}

\begin{abstract}
The model allows to calculate the amount of the costs to repair the defective parts of pipelines on the basis of statistical results. This model allows us to simplify the job for specialists in the industry.
\end{abstract}

\section{Keywords: Modeling, pipeline, forecasting.}

\section{INTRODUCTION}

Today, a wide variety of parameters are required to describe technological processes. As is known, the simulation process can be described quite accurately. In particular, to determine the number of defective sections of gas pipelines.

The authors of the article provide a simulation model for the development of corrosion defects using the Weibull distribution.

The model is based on the following conditions:

- defects grow and develop unevenly, therefore a set of defects is considered;

- the models take into account the date of the first defect for the corresponding type of pipe;

- considered the general appearance of defects [3, p. 22].

The main stages of creating a model:

1. development of a conceptual model;

2. preparation of initial data;

3. selection of modeling tools;

4. development of a software model;

5. checking the adequacy of the model;

6. Analysis of the results of modeling and decision making.

\section{BUILDING A SOFTWARE PACKAGE}

For the study of complex systems using a simulation model. It is presented in the form of software implemented blocks. With this method, you can create a simulation of both elementary phenomena and complex tasks. Using simulation, one can easily take into account the presence of discrete or continuous elements, random effects, etc.

The problem of generating a bandwidth with the Weibull distribution law is that the moment characteristics for this law are not expressed in terms of elementary functions [2]:

$$
\left\{\begin{array}{c}
m_{x}=\Gamma(1+1 / a) b^{-1 / a} \\
\sigma_{x}^{2}=\left[\Gamma(1+2 / a)-\Gamma^{2}(1+1 / a)\right] b^{-2 / a} .
\end{array}\right.
$$

Considering the direct problem of the distribution of the Weibull law, we can see that we can consider three models of the development of the defective area, depending on $m_{x}$ and $\sigma_{x}^{2}$

The primary goal of the authors was to predict with given parameters of expectation and variance, shape and scale parameters. This task is a difficult task in terms of forecasting, but has a fairly wide application for technological processes. For example, to determine the number of defective sections of gas pipelines.

The authors built a software package that allows you to get $m_{x}$ and $\sigma_{x}^{2}$ based on the Weibull distribution (Fig. 1.).

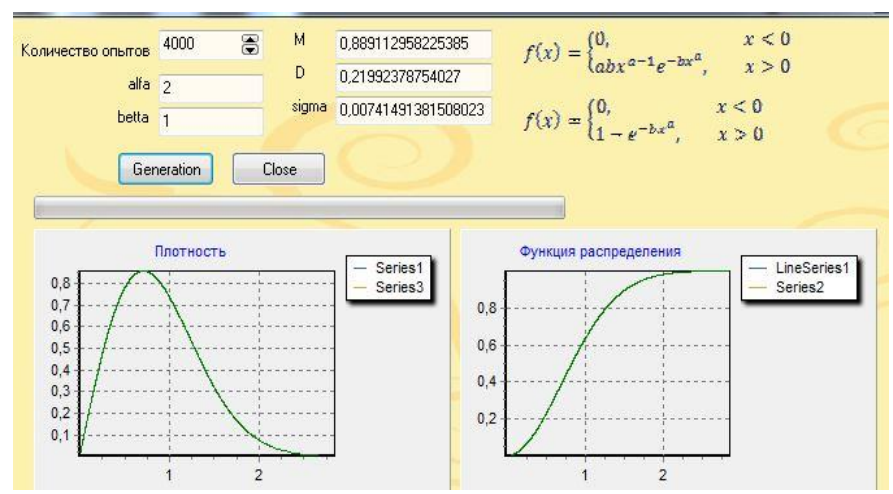

Fig. 1. The program for calculating the expectation, variance and error for a given Weibull distribution law

But to determine the operating time of a technical device, it is necessary to know which parameters $a$ and $b$ are necessary. In this regard, the task of creating a program that allows to calculate the inverse problem appears.

Thus, to generate a memory bandwidth with given $m_{x}$ and $\sigma_{x}^{2}$ it is necessary to solve the system of equations (1), i.e. determine the parameters of the Weibull distribution $a$ and $b$.

The decision was based on the following conditions:

if $m_{x} \geq 0,05 \quad b=16000 \cdot 16000^{-m_{x}}$,

$$
a=\frac{2,4 \cdot m_{x}}{0,7 \cdot \sigma_{x}^{2}} \cdot \frac{1}{34}+\sin \left(0,9 \cdot m_{x} \cdot \frac{\pi}{3}\right)-1,4 \cdot \ln \left(m_{x}\right)+3,5 ;
$$


if $0,01 \leq m_{x}<0,98$ and $0,0001<\sigma_{x}^{2}<0,027$

$$
b=\frac{\left(-\ln \left(\frac{m_{x}}{1,085}\right)\right)^{\frac{1}{8}}-0,8+\frac{e^{\frac{-11}{m_{x}}}}{3}+e^{\frac{-5 \cdot m_{x}}{4}}}{\sigma_{x}^{2}} .
$$

If $1 \leq m_{x}<1,8$, then

$$
a=\frac{3,2 \cdot m_{x}}{0,7 \cdot \sigma_{x}^{2}} \cdot \frac{1}{34}+\sin \left(1,4 \cdot m_{x} \cdot \frac{\pi}{4}\right)-1,4 \cdot \ln \left(m_{x}\right)+1,
$$

else $a=\frac{2,4 \cdot m_{x}}{\sigma_{x}^{2}} \cdot \frac{1}{34}+9 \cdot \sin \left(0,5 \cdot m_{x} \cdot \frac{\pi}{9}\right)+1,5 \cdot \ln \left(m_{x}\right)+3$.

\section{TABLE I. THE VALUES OF THE PARAMETERS OF THE} WEIBULL FUNCTION FOR B $=10$

\begin{tabular}{|c|c|c|c|c|}
\hline $\boldsymbol{a}$ & $m_{x}$ & $\sigma_{x}^{2}$ & $\mathrm{~b}^{*} \sigma_{x}^{2}$ & Estimated formula \\
\hline 2 & 0,2802 & 0,0214 & 0,2141 & 0,2069 \\
\hline 5 & 0,5794 & 0,0176 & 0,1758 & 0,1709 \\
\hline 10 & 0,7556 & 0,0083 & 0,0826 & 0,0827 \\
\hline 15 & 0,8285 & 0,0046 & 0,0457 & 0,0475 \\
\hline
\end{tabular}

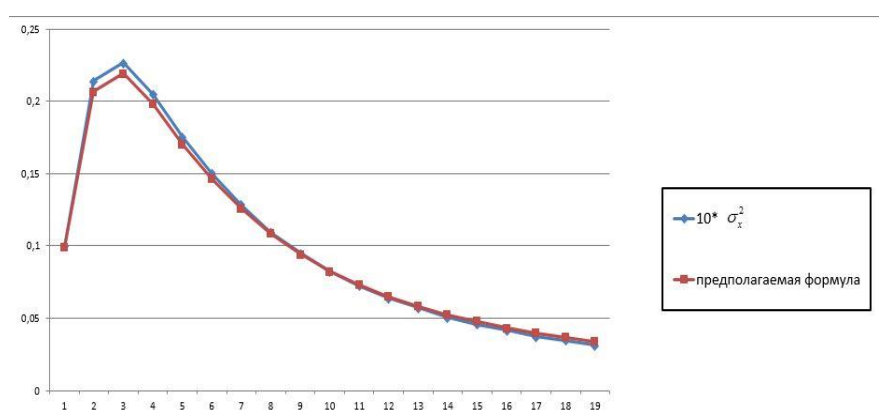

Fig. 2. The Weibull distribution function for the corresponding parameters $\mathrm{a}$ and $\mathrm{b}=10$

if $0,009 \leq m_{x}<0,96$ and $0,0001<\sigma_{x}^{2}<0,007$

k- coefficient

$$
k=\frac{-\ln \left(\sigma_{x}^{2}\right)}{4}
$$

if $k \leq 0,65$ then $b=\frac{k \cdot \sin \left(1,142857 \cdot 3,14 \cdot m_{x}\right)}{\sigma_{x}^{2}}$

else

$$
b=\frac{0,65 \cdot \sin \left(1,142857 \cdot 3,14 \cdot m_{x}\right)}{\sigma_{x}^{2}},
$$

$$
a=\frac{2,14 \cdot m_{x}}{\sigma_{x}^{2}} \cdot \frac{1}{34}+9 \cdot \sin \left(0,5 \cdot m_{x} \cdot \frac{\pi}{9}\right)+1,5 \cdot \ln \left(m_{x}\right)+3 .
$$

TABLE II. THE VALUES OF THE PARAMETERS OF THE WEIBULL FUNCTION FOR B $=100$

\begin{tabular}{|c|c|c|c|c|}
\hline $\boldsymbol{a}$ & $m_{x}$ & $\sigma_{x}^{2}$ & $\mathrm{~b}^{*} \sigma_{x}^{2}$ & $\begin{array}{c}\text { Estimated } \\
\text { formula }\end{array}$ \\
\hline 2 & 0,0879 & 0,0021 & 0,2119 & 0,2432 \\
\hline 5 & 0,3669 & 0,0069 & 0,6918 & 0,6973 \\
\hline 10 & 0,5999 & 0,0051 & 0,5176 & 0,6004 \\
\hline 15 & 0,7105 & 0,0034 & 0,3425 & 0,4086 \\
\hline
\end{tabular}

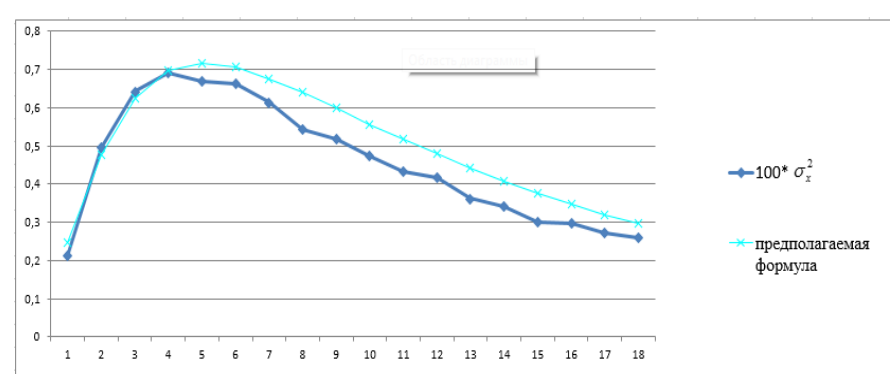

Fig. 3. Weibull distribution function for the corresponding parameters $\mathrm{a}$ and $\mathrm{b}=100$

In particular, the value of the coefficient $b=2$ was taken for this study on the distribution of defects. The values of the coefficients $\mathrm{a}$ and $\mathrm{b}$ are taken in accordance with the expected expectation and variance. The above formulas were used to determine the basic predictive course of the model. For extensive use only the first coefficient is changed.

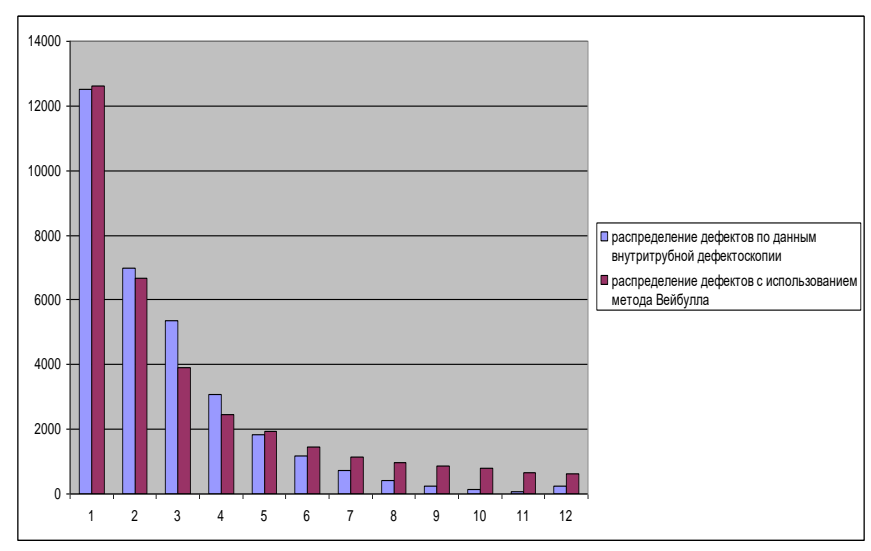

Fig. 4. Distribution of Corrosion Defects by Simulation Results

The distribution of defects according to the in-line defectoscopy, the authors took from [3].

Thus, the imitation system acts as a test system, allowing to achieve values close to the real model.

\section{REFERENCES}

[1] S.A. Prokhorov, M.S. Danilenko, "Application of the Weibull distribution law in the simulation of pseudo-random sequences", New Science: Strategies and Vectors of Development, vol. 4, 2015, pp. 8-11.

[2] M.S. Danilenko, "Solution of N-dimensional integrals by the MonteCarlo method", New science: Strategies and vectors of development, vol. 1-2(58), 2016, pp. 64-66.

[3] S.V. Nefedov, A.Y. Prokopenko, "Corrosion damage prediction model for trunk gas pipelines and a method for predicting the growth in the length of corrosive gas pipeline sections", Village Razvilka, Leninsky 
district, Moscow region: Publishing house Research and Development Institute of Natural Gases and Gas Technologies Gazprom VNIIGAZ, 2014, vol. 1 (17).

[4] S.A. Prokhorov, M.S. Danilenko, "Mathematical model of the dependence of the strength of wood on the density using the Weibull distribution function", Natural and technical sciences, 2017, vol. 1(103), pp. 139-144.

[5] S.A. Prokhorov, M.S. Danilenko, "Software package for simulating pseudo-random sequences using the Weibull distribution law", Economics and management management systems, 2016, vol. 21(3), pp. 94-100. 